\title{
METODOLOGIA ATIVA DE ENSINO-APRENDIZAGEM EM OPERAÇÕES DE MANUFATURA: ABORDAGENS DA TEORIA DAS RESTRIÇÕES POR MEIO DO USO DO OPT GAME
}

\section{ACTIVE METHODOLOGY OF TEACHING-LEARNING IN MANUFACTURING OPERATIONS: APPROACHES OF THE THEORY OF CONSTRAINTS THROUGH THE USE OF THE OPT GAME}

\author{
Elisandro João De Vargas* E-mail: elisandrovargas@gmail.com \\ Jocimara de Lima Mauer** E-mail: jocimara mauer@hotmail.com \\ Marcelo Alves de Souza** E-mail: msouzaadm@yahoo.com.br \\ Jayme Diego Silva Peixoto** E-mail: idspeixoto@gmail.com \\ Davenílcio Luiz de Souza** E-mail: davenilcio@gmail.com \\ *Instituto Federal de Educação, Ciência e Tecnologia Farroupilha \\ ** Universidade do Vale do Rio dos Sinos (UNISINOS), São Leopoldo, RS
}

Resumo: É importante a adoção de meios que facilitem aos alunos construírem seu conhecimento teórico e prático em assuntos relacionados a operações de manufatura. Nesse sentido, o objetivo deste artigo é apresentar o uso da metodologia ativa de ensino-aprendizagem na compreensão das abordagens da Teoria das Restrições (TOC) em operações de manufatura. Por meio de simulação com o uso do OPT Game, são apresentadas conexões entre o processo da TOC com os indicadores globais e operacionais, as regras do OPT, a sincronização da produção e o tambor-pulmão-corda. Caracteriza-se uma pesquisa aplicada, exploratória, descritiva e explicativa, por meio da técnica de simulação. O OPT Game foi aplicado em uma turma de mestrandos. Os resultados demonstraram que os alunos se sentiram motivados com a atividade, auxiliando-os na compreensão entre teoria e prática das abordagens da TOC; do qual foi considerado importante recurso facilitador à aprendizagem.

Palavras-chave: Metodologia Ativa de Ensino-Aprendizagem. Operações de Manufatura. Teoria das Restrições. OPT Game.

Abstract: It is important adoption means that facilitate the students to build their theoretical and practical knowledge in matters related to manufacturing operations. In this sense, the objective of this article is to present the use of the active methodology of teaching-learning in the understanding of the Theories of Constraints (TOC) approaches in manufacturing operations. Through simulation with the use of OPT Game, connections between the TOC process with the global and operational indicators, the OPT rules, the production synchronization and the drum-buffer-rope are presented. It is characterized an applied, exploratory, descriptive and explanatory research, through the simulation technique. The OPT Game was applied to a group of masters degree students. The results showed that the students felt motivated with the activity, helping them to understand the theory and practice of TOC approaches; whose was considered an important resource to facilitate learning.

Keywords: Active Teaching-Learning Methodology. Manufacturing Operations. Theory of Constraints. OPT Game. 


\section{INTRODUÇÃO}

É necessário adotar meios que instigue e auxilie o aluno a construir seu conhecimento. Afinal, refletir às formas de construir o conhecimento é um desafio que deve ser considerado no presente e no futuro entre docentes e discentes (VARGAS et al., 2016). Pesquisas demonstram a preocupação dos professores no ensino de operações em adequar o uso de cenários que possam auxiliar a gerar maior conhecimento e interesse nos estudantes (BOUYER; SZNELWAR; COSTA, 2005). Este fato tem gerado interesse em algumas instituições acerca do ensino na Engenharia de Produção (SANTOS; ARNAUD; DUTRA, 2014); à enfrentar o desafio de se adaptar às novas exigências de formação profissional, por consequência, de ensinar (FERNANDES BRUM; PURCIDONIO; FERRERA, 2017); como é o caso em operações de manufatura, a exemplo do aprendizado em abordagens da TOC.

Entre os motivos que despertam o interesse encontra-se a mudança do perfil dos estudantes e os requisitos do mercado de trabalho (SANTOS; ARNAUD; DUTRA, 2014). A mudança no perfil, por exemplo, nos estudantes de graduação; atualmente possuem a sua disposição amplo acesso à obtenção de informações e tecnologias. Além disso, o mercado de trabalho requer a atuação de profissionais com competências para a solução de problemas do mundo real. Para Carmo, Barroso e Albertin (2010), é necessária a formação de profissionais, entre eles os engenheiros, com habilidades e capacidades de análise crítica acerca de situações além da capacidade técnica.

Uma das maneiras de incrementar o ensino é o uso da metodologia ativa de ensino-aprendizagem, da qual busca promover mudanças sistêmicas em relação a direção à pesquisa, operações, e a própria educação (WALS, 2014). Esta metodologia enfatiza no aluno o foco principal na construção do próprio conhecimento, em vista a conexões entre os conteúdos (BONWEL; EISON, 1991). Neste sentido, a formação de um profissional ultrapassa as barreiras do simples entendimento para a compreensão por meio da aplicação prática e interpretação dos resultados gerados, ou seja, do entendimento teórico para a compreensão prática.

Com o uso de tecnologias computacionais é possível representar ambientes artificiais, criar eventos, simular e testar inúmeras situações e explorar novas estratégias rapidamente no tempo e no espaço, permitindo aos estudantes e 
profissionais a compreensão dos fatos (FIGUEIREDO, 2010). Tais relações são fundamentais na Engenharia de Produção para o desenvolvimento de práticas e a melhorar a gestão e modernização dos ambientes empresariais.

Entre os assuntos abordados no ensino de operações de manufatura estão os conceitos relacionados a TOC. A TOC visualiza a empresa como um sistema composto de relações interdependentes que determinam 0 resultado global (FIGUEIREDO; PEREIRA; VASCONCELOS, 2013). Sua abordagem expande a fronteira além do sistema produtivo com vistas ao gerenciamento da empresa como um todo (RODRIGUES, 1990). Nas abordagens da TOC é possível encontrar o processo de focalização da TOC, os indicadores globais e indicadores operacionais, as regras do OPT, sincronização da produção e o tambor-pulmão-corda.

A compreensão destas abordagens é de extrema importância, haja vista a necessidade das empresas em atingir resultados que as direcione à sua meta. Dessa maneira, as abordagens da TOC podem ser melhor entendidas quando inseridas em um ambiente de simulação, com a participação ativa do aluno na construção de seu aprendizado (FIGUEIREDO, 2010). Por exemplo, o uso do OPT Game possibilita simular o encadeamento das abordagens da TOC. Sua criação ocorreu pela Creative Output, empresa de Eliyahu M. Goldratt, visando demonstrar os conhecimentos expostos nas palestras de Goldratt; ilustrando o cotidiano das empresas em suas atividades de manufatura.

Neste intuito, este artigo visa contribuir na explanação acerca do uso da metodologia ativa com base em simulação no ensino de operações de manufatura. O objetivo principal é demonstrar o uso do OPT Game como recurso facilitador no entendimento e compreensão das abordagens da TOC sob o olhar da metodologia ativa de ensino-aprendizagem. Os objetivos específicos são: (i) demonstrar por meio de ilustrações o uso do OPT Game; (ii) gerar conexões entre o uso do OPT Game e as abordagens da TOC relativas ao processo de focalização da TOC, os indicadores globais e indicadores operacionais, as regras do OPT, sincronização da produção e o tambor-pulmão-corda; e, (iii) apresentar os resultados de um estudo aplicado.

Os resultados do estudo aplicado demonstraram que os alunos se sentiram motivados em realizar a atividade/ jogo; auxiliando-os no entendimento e compreensão de algumas das abordagens da TOC; considerando importante recurso à aprendizagem. 
O artigo está estruturado em: referencial teórico, metodologia, ilustração do uso do game, caso aplicado e considerações finais.

\section{REFERENCIAL TEÓRICO}

\subsection{Metodologia Ativa de Ensino-Aprendizagem e a Conjuntura no Ensino de Operações de Manufatura}

Novas formas de aprendizagem e abordagem denominadas Aprendizagem Ativa surgem, tendo por foco promover mudanças sistêmicas em relação a direção à pesquisa, operações, e a própria educação (WALS, 2014). Na concepção de Bonwell e Eison (1991), a metodologia ativa de ensino-aprendizagem refere-se as estratégias de ensino em que proporciona ao aluno maior conquista de autonomia em seu processo de aprendizagem. O aluno colabora com conexões entre conteúdos novos e antigos, fomentando maior participação na construção de sua própria aprendizagem.

Na década de 60, Bruner (1961) mencionava a importância do conhecimento prévio no processo de aprendizagem, em que os alunos envolvidos de maneira ativa com o conteúdo estudado são mais propensos a recordarem das informações recebidas por mais tempo. Para Barbosa e Moura (2013), os alunos necessitam ser estimulados a construir o seu conhecimento ao invés de receber o conteúdo de maneira passiva. Para os autores, o professor intervém como mediador, orientador ou supervisor que contribui no processo de aprendizagem. Para Vigotsky (2010), por exemplo, o professor do ensino superior necessita ser um agente mediador no processo de ensino-aprendizagem, promovendo desafios aos acadêmicos.

Neste sentido, o objetivo central em abordagens da metodologia ativa de ensino-aprendizagem é a aprendizagem do aluno, em que a importância do aprender prevalece o de ensinar. Segundo Santos, Arnaud e Dutra (2014), no ensino da Engenharia de Produção, esta metodologia ativa de aprendizagem vem acontecendo com frequência. Afinal, para Carmo, Barroso e Albertin (2010), é necessária a formação de profissionais, entre eles os engenheiros, em habilidades e capacidades de análise crítica acerca de situações além da capacidade técnica. 
Com o advento das ferramentas computacionais, é possível que simulações sejam efetuadas, ambientes criados e 0 tempo e espaço comprimidos (FIGUEIREDO, 2012). A compressão possibilita a análise dos efeitos colaterais ao longo do tempo, identificar os erros e formular novas estratégias. Isso permite que os participantes sejam os atores principais, mantendo sob seu controle conceitos, procedimentos e fatos, gerando aprendizado.

Eliyahu M. Goldratt (1947 - 2011) propôs a utilização de vários instrumentos funcionais, como jogos que demonstram a variabilidade e influência nos sistemas produtivos, livros em forma de romance exibindo os temas centrais de sua teoria, vídeos educacionais, por exemplo "A Meta" e softwares educacionais como é o caso do OPT Game (ANTUNES, 1998). De acordo com Goldratt (2013), o OPT Game é um jogo com certo grau de dificuldade, do qual sugere que os alunos operem em pares, com a possibilidade de jogar a qualquer momento sem a necessidade de supervisão. Conforme os autores, o jogo aborda várias das considerações existentes no livro A Meta (1984), possibilitando ao jogador aplicar várias abordagens da TOC. Ao final, alguns princípios ficam fixados em suas mentes, principalmente em relação a importância da gestão dos gargalos para a obtenção da produtividade e lucro.

Por fim, salienta-se existir distintas formas de ser aplicada a metodologia ativa de aprendizagem, das quais pode-se citar: a transdisciplinar e interdisciplinar, social, com base em projetos, em jogos de simulação, em simulação computacional, via o desenvolvimento de cenários, com base em problemas, dentre outras. Neste artigo foi explorada a metodologia de jogos de simulação.

\subsection{Do OPT (Optimized Production Technology) à TOC (Teoria das Restrições)}

Uma importante diferença entre o pensamento OPT e o software OPT, é que o primeiro abrange os princípios e ideias da técnica e o segundo formaliza essas ideais em um sistema computacional como forma de gerenciar os "gargalos" (RODRIGUES, 1990). Gargalo é todo e qualquer recurso cuja demanda imposta é maior que sua capacidade (GOLDRATT; COX, 2011), sendo poucos ou apenas um, normalmente por um espaço de tempo considerável (ANTUNES et al., 2008).

A Teoria das Restrições visualiza a empresa como um sistema composto de relações interdependentes que determinam o resultado global (FIGUEIREDO; 
PEREIRA; VASCONCELOS, 2013). Pode ser entendida como uma metodologia sistêmica de construção e solução de problemas (MABIN; BALDERSTONE 2003), além de apresentar perspectiva de olhar sistêmico integrado (GUPTA; BOYD, 2008). Esta perspectiva expande a fronteira para além do sistema produtivo, contemplando o gerenciamento da empresa como um todo, passando da palavra gargalo para restrição (RODRIGUES, 1990).

Foi por solicitação de um amigo no início dos anos 70 que Elyahu M. Goldratt desenvolveu um sistema de programação denominado OPT, comercializando-o por meio de sua empresa Creative Output em Israel e filiais em outros países (RODRIGUES, 1990). A partir de 1985 o software se utilizou do conceito de "pulmão de tempo" (GOLDRATT, 1988), pela introdução de vários princípios que vieram a construir o pensamento OPT (RODRIGUES, 1990).

Assim, em 1984 em conjunto com Jeff Cox, Goldratt editou o livro A Meta, apresentando em forma de romance a aplicação prática dos princípios do OPT (GOLDRATT; COX, 2011). Além disso, Goldratt promoveu várias palestras em universidades americanas e europeias divulgando suas técnicas, e ao mesmo tempo, visando elucidar os princípios do qual abordava, sua empresa Creative Output desenvolveu um simulador denominado OPT Game, possibilitando que as pessoas vivenciassem os problemas cotidianos de uma fábrica e interagissem na prática às dificuldades no gerenciamento de uma empresa (RODRIGUES, 1990).

Em 1985 o software OPT e os direitos da marca foram vendidos para a empresa Sheduling Technologies Group Limited. A partir deste momento, Goldratt avança em termos de ideias surgindo a TOC, ampliando o pensamento OPT, passando a ser visto como vendedor de ideias, com maior enfoque na TOC. (RODRIGUES, 1990). A TOC olha a empresa como um sistema composto por elementos dos quais possuem alguma relação de interdependência, situação em que o desempenho depende dos esforços de todos os elementos do sistema (FIGUEIREDO, 2010). Este processo de pensamento pode contribuir para o aprimoramento constante das organizações em diversas áreas (FILHO et al., 2012).

O processo de pensamento da TOC é um conjunto de técnicas analíticas fundamentadas no método científico, as quais embasadas neste método permite à identificação, à análise e solução de problemas, além do uso da dialética no desenvolvimento do método geral (ANTUNES, 1998). Estas técnicas permitem 
serem utilizadas individualmente ou encadeadas logicamente (COX; SPENCER, 2008); possibilitando um olhar sistêmico integrado (GUPTA; BOYD, 2008). Neste artigo são analisados: o processo de focalização da TOC, os indicadores globais e indicadores operacionais, as regras do OPT, sincronização da produção e o tamborpulmão-corda.

\subsubsection{Processo de focalização da TOC}

O processo de focalização da TOC é formado por cinco etapas que visualizam a empresa de maneira global, embasada pelo processo de melhoria contínua (GOLDRATT; COX, 2011). Estes passos podem servir para identificar e gerenciar o gargalo do sistema, condição importante para o gerenciamento da capacidade produtiva (PACHECO et al., 2012). A Tabela 1 apresenta a sequência de passos da focalização da TOC.

Tabela 1 - Focalização da TOC

\begin{tabular}{|rl|}
\hline 1. & identifique a(s) restrição(ões) do sistema. \\
\hline 2. & explore a(as) restrição(ões) do sistema. \\
\hline 3. & subordine tudo a decisão da etapa anterior. \\
\hline 4. & eleve a restrição do sistema. \\
\hline 5. & se mudar a restrição, retorne a etapa um, não deixando a inércia tornar a restrição do sistema. \\
\hline
\end{tabular}

Fonte: Goldratt e Cox (2011) e Antunes Jr. (1998)

Os passos serão abordados durante a simulação, e são fundamentais para o aprendizado da TOC. Caso os passos sejam ignorados ou seguidos de forma incorreta, podem afetar os resultados da simulação, assim como na aplicação prática da teoria nas empresas.

\subsubsection{Indicadores globais e operacionais da TOC}

A TOC possui indicadores operacionais e globais. De acordo com Goldratt e Cox (2011), a meta da empresa é ganhar dinheiro hoje e no futuro. Para controlar o rumo da meta da empresa satisfatoriamente, a meta é associada a indicadores auxiliando na análise qualitativa e quantitativa, desdobrando-se em indicadores globais e indicadores operacionais (ANTUNES JR., 1998). Haja vista que a forma de 
medir e os tipos de medir podem auxiliar a identificar se está no rumo à meta projetada.

Sobre isso, Goldratt (1991, p. 28) diz: "diga-me como me medes e te direi como me comportarei, se me medires de forma ilógica não reclame de comportamento ilógico". A alusão feita por Goldratt expressa claramente a importância em apresentar coerência entre as medições. No caso, os indicadores globais são considerados de nível mais estratégico; já, os indicadores operacionais são considerados de nível mais gerencial. Notoriamente, os indicadores operacionais e globais apresentam correlações, conforme indicado na Tabela 2.

Os indicadores são expressos por Goldratt e Cox (2011), Rodrigues (1990), Goldratt (1991) e Antunes et al. (2008) em:

Tabela 2 - Indicadores da TOC

\section{Indicadores Globais}

\begin{tabular}{|c|c|}
\hline Lucro Líquido & $\begin{array}{l}\text { Relaciona-se ao resultado entre o "ganho - despesa operacional". Representa o total de } \\
\text { dinheiro gerado pela empresa, medida absoluta, distinta do conceito de lucro líquido } \\
\text { contábil; }\end{array}$ \\
\hline $\begin{array}{l}\text { Retorno sobre o } \\
\text { Investimento }\end{array}$ & $\begin{array}{l}\text { Resultado entre "ganho - despesa operacional / inventário". Considerado uma medida } \\
\text { relativa da qual indica o esforço para atingir certo nível de lucro; }\end{array}$ \\
\hline Fluxo de Caixa & $\begin{array}{l}\text { Representa a saúde financeira da empresa, neste sentido "se há caixa, então o caixa não é } \\
\text { importante, no entanto, se não há caixa, ele é o mais importante". Sua importância ganha } \\
\text { força principalmente em ambientes cujas taxas de juro são maiores. }\end{array}$ \\
\hline \multicolumn{2}{|r|}{ Indicadores Operacionais } \\
\hline Ganho & $\begin{array}{l}\text { Proporciona a entrada de dinheiro no sistema. É o resultado da produção que se } \\
\text { transforma em vendas. Seu resultado se obtém entre "valor líquido de vendas - o valor de } \\
\text { matéria-prima". }\end{array}$ \\
\hline Inventário & $\begin{array}{l}\text { Dinheiro investido, sejam em máquinas, estoques, equipamentos, entre outros; do qual o } \\
\text { propósito esteja relacionado em transformar em vendas no futuro. }\end{array}$ \\
\hline $\begin{array}{l}\text { Despesas } \\
\text { Operacionais }\end{array}$ & $\begin{array}{l}\text { Representa o dinheiro gasto na transformação do inventário em ganho, por exemplo, os } \\
\text { trabalhos diretos e indiretos. }\end{array}$ \\
\hline
\end{tabular}

Fonte: Adaptado de Goldratt e Cox (2011), Rodrigues (1990), Goldratt (1991) e Antunes et al. (2008)

Para Goldratt (1991) e Goldratt e Cox (2011), o ganho deve ser considerado o principal elemento a ser obtido, posteriormente o inventário por refletir no ganho, e por último a despesa operacional. Este ponto de vista é considerado a lógica do "mundo dos ganhos". Este mundo procura proteger o ganho pela proteção do elo mais fraco, do qual determina a "resistência da corrente" pela realização de melhorias na restrição (GOLDRATT, 1991). Neste sentido, a maximização dos resultados da empresa pode ser ampliada por meio da identificação da restrição e a 
determinação do ganho versus o tempo de utilização da restrição, subordinando as demais atividades sob a cadência da restrição, minimizando ao máximo os estoques não necessários (LACERDA; RODRIGUES, 2007).

Por outro lado, o "mundo dos custos" objetiva controlar o custo, por exemplo, pela redução dos custos alocados ao produto (LACERDA; RODRIGUES, 2007). Sua lógica considera como prioridade as despesas operacionais, posteriormente 0 ganho, e por último o inventário (GOLDRATT, 1991).

\subsubsection{Regras do OPT}

Goldratt e Cox (2011) fazem algumas indagações a serem respondidas: (i) qual a meta de uma empresa e quem a determina?; (ii) como saber se a meta da empresa está sendo alcançada?; e, (iii) como determinar ações locais que auxiliem ao alcance do ótimo global? As respostas às perguntas podem auxiliar no desenvolvimento da programação e sequenciamento da produção, ao mesmo tempo, compreender a máxima "a soma dos ótimos locais não é igual ao ótimo global (GOLDRATT; COX, 2011)".

Visando auxiliar nas respostas às perguntas, Goldratt formalizou às nove regras que visam a sincronização da produção, as quais podem auxiliar a atingir a meta da empresa (RODRIGUES, 1990; BLACKSTONE, 2013). De acordo com Goldratt (1988), as regras são:

Regra 1: balancear o fluxo, não a capacidade: balancear o fluxo apresentará melhores resultados, considerando a dificuldade de equilibrar a capacidade. Para isso, a capacidade do posto anterior deverá ser menor que a restrição;

Regra 2: o nível de utilização de um não gargalo não é determinado por seu potencial, mas pela restrição do sistema: o ritmo do posto de menor capacidade, denominado de restrição, é que determina o não gargalo, de mesmo modo, é a eficiência da restrição que determina a eficiência do não gargalo;

Regra 3: utilização e ativação de um recurso não são sinônimos: ativar um recurso só é válido se gerar valor para a empresa, ou thoughput que potencializem sua utilização; 
Regra 4: uma hora perdida em um gargalo é uma hora perdida no sistema como um todo: gargalo é o recurso sem sobra de capacidade cuja utilização já ocorre 24 horas e sete dias por semana; neste caso, qualquer tempo perdido no gargalo reflete em todo o sistema na geração de thoughput;

Regra 5: uma hora economizada em um recurso não gargalo é apenas uma miragem: haja vista que este recurso possui capacidade incremental, poderá se recompor por algum eventual problema não influenciando no thoughput;

Regra 6: os gargalos determinam tanto os ganhos quanto o estoque: os gargalos limitam às saídas no sistema, os estoques sequentes e os ganhos decorrentes de sua produção;

Regra 7: o lote de transferência pode não ser, e em muitos casos não deveria ser, igual ao lote de processamento: considerando que quanto menor o lote de transferência (entre estações), em detrimento ao lote de processamento (itens processados entre preparações); irá incidir em execução simultânea de um mesmo pedido, reduzindo o tempo de atravessamento e o estoque em processo;

Regra 8: os lotes de processamento devem ser variáveis e não fixos: a dinâmica do mercado, sazonalidades, novos produtos, entre outros eventos podem influenciar na composição dos lotes entre os diferentes meses;

Regra 9: as programações devem ser realizadas examinando simultaneamente todas as restrições e os tempos de atravessamento não decorrentes da programação, dos quais não podem ser pré-determinados: isso ocorre pela existência do agrupamento de diversas variáveis que afetam o sistema, ocasionando como resultado um recurso que prevalecerá como sendo o mais crítico.

\subsubsection{Tambor-Pulmão-Corda (TPC)}

O TPC se desenvolveu pelas experiências de Goldratt com seu software OPT, ao identificar que nem todos os equipamentos necessitavam ser utilizados $100 \%$ do tempo (GOLDRATT, 1988). É considerado um princípio elementar da TOC, em que busca otimizar o sistema de produção conforme a capacidade de cada recurso (SOUZA, 2005). Caso os postos de trabalho possuam capacidade superior à demanda, estoques devem ser evitados (BLACKSTONE, 2013). De acordo com Goldratt (1991) e Srikanth (2013), o TCP pode ser entendido em: 
a) Tambor: representa o recurso com restrição de capacidade (CCR). Este recurso CCR determina a cadência dos demais recursos e a capacidade total produtiva no sistema;

b) Pulmão: corresponde a proteção do sistema para possíveis incertezas que gerem risco para o recurso CCR. Esta ação minimiza os impactos da variabilidade no sistema;

c) Corda: é o mecanismo de promover a sincronização entre a entrada dos materiais no pulmão (estoque protetor) e a entrada de materiais no sistema, visando mínimos estoques intermediários e programação da produção puxada.

O CCR corresponde aos recursos com restrição de capacidade, que estão no limiar para se tornarem gargalos, necessitando de atenção e aprimoramento juntamente com os recursos gargalos (GOLDRATT; COX, 2011). O limiar é motivado pela variabilidade dos sistemas e flutuações de demanda que, em média, apresentam capacidade superior à demanda (ANTUNES JR, et al., 2008).

De acordo com Blackstone (2013), são poucos os CCR existentes em uma fábrica, denominados de "tambor". Assim, para evitar que o CCR pare são gerados estoques de materiais, denominados de "pulmão". Estes estoques são controlados suas entradas no recurso restritivo por meio da "corda".

\section{METODOLOGIA}

O presente artigo utiliza do método de modelagem pela técnica de simulação, de caráter aplicado, exploratório, descritivo e explicativo, seguindo as premissas de MIGUEL et al., (2012). Neste sentido, está organizado visando gerar conhecimento no decorrer de sua aplicação, apresentando situações dinâmicas e interativas. Ao mesmo tempo, utiliza de teorias e leis que visam auxiliar na explicação dos fenômenos. Em um primeiro momento explica as ideias, passando a descrição de características acerca dos eventos e fatores que sustentam a ocorrência dos eventos.

O desenvolvimento do jogo proposto utiliza o referencial teórico abordado, do qual é introduzido em meio as explicações dos comandos e das variáveis e regras do jogo. Com isso, é apresentada as etapas, conexões e encadeamento da 
execução do jogo, visando conduzir o leitor a operar o jogo, promover entendimento e compreensão.

O estudo aplicado exibe resultados e considerações dos alunos mestrandos em Engenharia de Produção, período 1/2015, de uma universidade Z, que utilizaram o OPT Game na elucidação de conhecimentos sobre abordagens da TOC. Foram organizadas equipes de três alunos, com o tempo de duas semanas após a explicação teórica dos assuntos da TOC para exercitar o jogo OPT Game e desenvolver um relatório mencionando as principais abordagens da TOC utilizada e/ou outros recursos que viabilizaram chegar ao máximo de ganho. Também, a responder as perguntas: i) qual o mix a ser produzido; (ii) quanto e quando produzir; e, (iii) como produzir. Em aula posterior às duas semanas, foi conduzida a apresentação dos resultados obtidos de cada equipe, dúvidas e questionamentos entre discentes e docentes.

Após a conclusão da atividade em julho/15, os alunos foram convidados a responder um questionário com sete perguntas fechadas e uma aberta. Ao total, participaram 15 alunos e foi obtido o retorno de 11 alunos. As perguntas fechadas foram organizadas em uma escala Likert de 1 a 5 , em que 1 representa "discordo plenamente" e 5 "concordo plenamente"; conforme resultados apresentados na Tabela 3. A pergunta aberta buscou identificar sobre "Na sua opinião, quais foram os pontos positivos e negativos, facilidades e dificuldades encontradas no desenvolver do jogo?". Destaca-se que a atividade realizada não utilizou da contextualização entre jogo e teoria, conforme contido neste artigo. Os resultados são apresentados e argumentados na subseção 4.3.

\section{O JOGO OPT GAME E AS RELAÇÕES COM AS OPERAÇÕES DE MANUFATURA}

O OPT Game era disponibilizado por Goldratt ao final de suas palestras com o objetivo dos participantes exercitarem os conhecimentos por ele apresentado (ANTUNES et al., 2008). Mesmo se tratando de um jogo com anos de criação, o OPT Game consegue representar os desafios vivenciados por gestores, técnicos e analistas, ilustrando tipicamente algumas das decisões necessárias dentro das empresas em suas atividades de manufatura. 
Assim como nas empresas, o objetivo do OPT Game é chegar ao final do jogo com o máximo de resultado, ou seja, lucro. Para isso, inicialmente é necessário conhecer as regras do jogo, as premissas básicas de funcionamento que regem a simulação. Analogamente, em uma empresa é necessário conhecer suas premissas, objetivos e formas de funcionamento que movimentam o sistema. É necessário conhecer o mercado, as entradas, os meios de transformação e as saídas.

Em suma, o enfoque do jogo visa identificar: (i) qual a estratégia a ser utilizada que maximize os lucros considerando os custos de MP, os tempos e os valores de venda dos produtos; (ii) qual ou quais as restrições existentes no sistema; e, (iii) quais as estratégias a serem adotadas em termos de programação e sequenciamento que informem o mix, o quanto e como deve ser produzido. Neste sentido, o desenrolar do jogo utiliza das diversas abordagens da TOC afim de identificar estes questionamentos.

\subsection{As ferramentas e regras do jogo}

$O$ jogo exige instalação em equipamento com versão Windows $X P$. O comando é executado por teclado e as orientações e informações são em língua inglesa. Acerca da versão Windows XP, é possível efetuar a virtualização da máquina/computador, possibilitando que seja instalada em qualquer versão e/ou plataforma, neste caso, a versão recomendada para o jogo. Sugestivamente, muitas maneiras práticas podem ser obtidas na internet. Para mais detalhes acerca de virtualização ver Carissimi (2008). O comando por teclado é prático e as orientações e informações acerca do jogo podem ser utilizadas conforme este artigo.

Ao iniciar o jogo estará disponível cinco opções ao usuário: 1. iniciar o jogo; 2. autoajuda; 3. designar novos parâmetros para o jogo; 4. executar jogo com os novos parâmetros; 5. sair do jogo.

As regras a serem atendidas durante a simulação são (MULLER et al., 1995):

a) O caixa será iniciado com a restrição de valor em $\$ 1500,00$. Este valor pode ser utilizado para a compra das matérias-primas ou cobrir parte das despesas fixas semanais; 
b) Ao final de cada semana é necessário pagar as despesas fixas no valor de $\$ 2.500,00$, portanto, é necessário que você possua dinheiro em caixa o suficiente para "não quebrar";

c) $O$ jogo tem duração de 6 semanas, considerando 5 dias por semana e 8 horas de trabalho por dia;

d) Os preços da MP RM1 e RM2 é de \$10,00 e os preços de venda de P1 \$ $30,00, P \$ 60,00$ e $P 2 \$ 30,00$;

e) Para qualquer produto vendido de $\mathrm{P} 1$ e P2, possui como restrição a necessidade da venda da mesma quantidade de produto $P$;

f) O produto $P$ é formado por 1 unidade da MP RM1 e RM2, o produto P1 é formado 1 unidade da MP RM1 e o produto P2 é formado 1 unidade da MP RM2.

g) Não há restrição de demanda, tudo o que for produzido poderá ser vendido, sua restrição será interna;

h) Cada máquina possui um tempo de setup que está disposto no lado esquerdo, e um tempo de processamento por unidade disposto no lado direito da figura da máquina em minutos, conforme a Figura 1;

i) Existe a restrição de operação entre algumas máquinas, onde deverá ser escolhida uma operação em detrimento a outra, que é o caso de $\mathrm{A}$ ou $\mathrm{C} \mathrm{e}$ $B$ ou $D$, que representam diferentes máquinas, Azul, Verde ou Vermelha. Nada impede que você interrompa uma operação e inicie em outra, mas lembre-se, a cada mudança o equipamento necessitará de preparação (setup). As informações podem ser visualizadas conforme a Figura 1. 
Figura 1 - Tela do jogo OPT Game

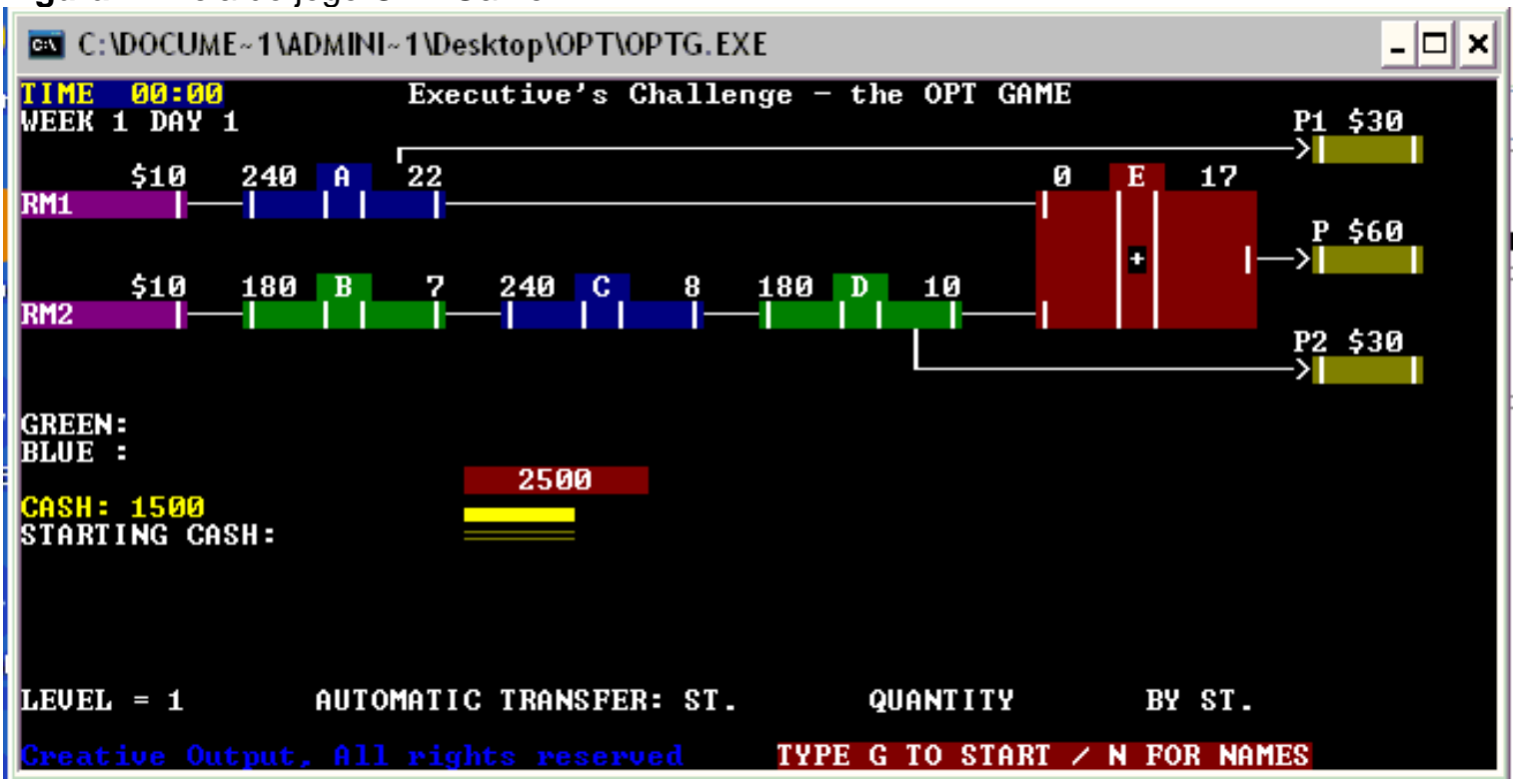

Fonte: Muller et al. (1995)

As instruções do painel de comando do jogo são apresentadas no Quadro 1.

Quadro 1 - Instruções do painel de comando do OPT Game

Setas: "direita, esquerda, cima, baixo" ou comando

曰 A-E direcionam "você" entre as operações/ equipamentos; clicando "enter" dará o comando de compra de MP, início de setup ou transferência de material.

G: inicia o jogo ou continua o processo de onde parou.

F: parar o jogo.

R: reinicia o jogo.

T: proporciona programar uma transferência de material entre as operações onde: ST é a estação de que transfere, QUANTITY é a quantidade desejada a transferir, atingindo este número é realizada a transferência, e BY ST é a estação que receberá a quantidade programada. Requer 10 minutos para realizar as transferências entre as estações.

S - Parar o jogo e salvar o contexto (para continuar em outro momento).

$\mathbf{Q}$ : termina o jogo.

F1: ao final de cada semana, quando for clicado em F1, é apresentado o estado de ativação de cada equipamento, representado pela letra da operação

CC o tempo de máquina em operação, e a parte

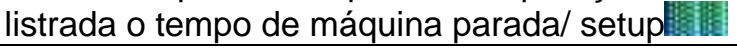

Level: maneira de reduzir ou aumentar a velocidade do jogo, ajustado pelos símbolos () "menos" e (+) "mais".

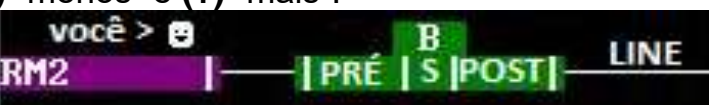

Compra de material: posicionar "você" sobre a caixa RM1 ou RM2 e digitar a quantidade e clicar em "enter".

Iniciar um setup: posicionar "você" sob a caixa "PRÉ" e clicar em "enter". Serve para qualquer operação, lembrando que "você" pode iniciar um setup a qualquer momento, porém há o tempo de preparação do equipamento.

Transferir produtos: posicionar "você" sob a caixa "POST" e clicar em "enter", ou utilizar o comando "T".

Direcionar as vendas: posicionar "você" sobre a "line" e clicar "enter".

As indicações dos sinais abaixo da operação: "S" indica estar em setup; " $\rightarrow$ " em processamento; "T" transferência automática; "+" aguardando processamento.

\subsection{Desenvolvimento do jogo}

Entendido os comandos e regras de funcionamento do jogo, é momento de praticar, exercitando algumas abordagens da TOC. 
Em alguns sistemas de operações de manufatura é possível identificar diversas atividades e equipamentos. Ainda é possível visualizar diferenças entre atividades e equipamentos, as quais normalmente são interdependentes determinando o resultado de uma empresa. De acordo com Figueiredo Pereira e Vasconcelos (2013), esta interdependência que gera determinado resultado global é um dos entendimentos que aborda a TOC.

Uma das diferenças comuns nos sistemas de manufatura são os tempos de processamento entre equipamentos e produtos. Estes tempos somados aos diferentes volumes de produção podem, por exemplo, provocar flutuações quanto ao uso dos equipamentos; podendo originar uma necessidade maior que a capacidade disponível do recurso, ou seja, um gargalo. Conforme Goldratt e Cox (2011), os gargalos surgem quando a demanda necessária é maior que a capacidade, o que requer gerenciamento adequado a este gargalo(os). Caso o gerenciamento expanda além das fronteiras da produção, este "gargalo" existente no sistema, do qual poderia estar no marketing, contabilidade, distribuição, outro; passaria a ser denominado de restrição (RODRIGUES, 1990). O uso do termo gargalo e restrição será utilizado neste artigo no sentido de recurso cuja capacidade está ou encontrase próximo do seu limite, que possa restringir o aumento de ganho do sistema.

Neste entendimento, no jogo, o primeiro passo necessário é identificar qual(quais) a(as) restrição(ões) no sistema. Para auxiliar a identificar a restrição é possível utilizar as cinco etapas de focalização da TOC. Similarmente, identificar a restrição de uma empresa permite que as ações possam ser direcionadas naquilo que possibilite melhorar o resultado.

No jogo OPT Game é possível criar uma tabela contendo os produtos, máquinas e tempos de processamento e setup. Seguindo as etapas de focalização da TOC, com a tabela criada, é possível identificar a restrição do sistema, ou seja, entre os produtos $\mathrm{P}, \mathrm{P} 1$ e $\mathrm{P} 2$, seus tempos de processamento e de setup para a máquina azul, verde e vermelha; é possível identificar qual equipamento será mais utilizado na fabricação dos produtos, ou tempos de setup são maiores. Passando a explorar a restrição, é possível vislumbrar ações que possam ser empregadas para que se obtenha o máximo de ganho na restrição, por exemplo, situação que necessitar realizar maior número de setup. $\mathrm{Na}$ terceira etapa, é necessário subordinar tudo a decisão da etapa anterior, isso representa organizar os eventos do 
sistema como um todo em função da etapa dois, visando ao mesmo tempo reduzir os inventários e despesas operacionais. A quarta etapa infere em elevar a restrição do sistema, no caso do OPT Game isso não é possível; em uma empresa, esta opção poderá ser viável, por exemplo, com horas extras, aumento no número de máquinas ou outra ação que aumente a capacidade do recurso restritivo. A quinta etapa vislumbra retornar a etapa um em decorrência às mudanças ocorridas no sistema como um todo. Conforme (GOLDRATT; COX, 2011), isso denota o processo de melhoria contínua.

No jogo, o segundo passo representa identificar entre os produtos às margens que cada um disponibilizará. Para isso, é possível realizar uma tabela contendo a relação entre os produtos $\mathrm{P}, \mathrm{P} 1$ e P2, preços de venda menos os preços das MP utilizadas. Visando maximizar o ganho, é necessário analisar às margens dos produtos em relação ao tempo de utilização de cada produto na máquina restritiva, do qual apresentará o $\$$ /minuto que cada produto irá contribuir na geração de ganho no recurso restritivo. Esta ação auxilia a identificar o direcionamento nas vendas entre os produtos $\mathrm{P}: \mathrm{P} 1, \mathrm{P}: \mathrm{P}, \mathrm{P} 2: \mathrm{P} 1, \mathrm{P} 1: \mathrm{P}: \mathrm{P} 2$.

O segundo passo busca proteger o ganho pela proteção do elo mais fraco, segundo (LACERDA; RODRIGUES, 2007), isso ocorre com a realização de melhorias na restrição, em que as demais atividades são subordinadas a cadência da restrição. Esta ação é denominada mundo dos ganhos, ou seja, caso utilize apenas o resultado da margem de cada produto, sem a relação da contribuição da margem versus a utilização no recurso restritivo; é possível que seja priorizado a fabricação de um produto que gere menor margem final para a empresa.

Em suma, no segundo passo é identificado o mix de produtos a serem produzidos. Neste sentido, no OPT Game é necessária atenção quanto aos produtos e quantidades que serão priorizadas em virtude das regras do jogo. Em uma empresa, o desdobramento entre indicadores qualitativos e quantitativos, análise global ou operacional, segundo Goldratt (1991) e Goldratt e Cox (2011), devem priorizar primeiramente o ganho, após redução de inventário e despesa operacional.

No terceiro passo é necessário elaborar o sequenciamento e programação da produção. No OPT Game isso ocorre na simulação de seis semanas, sendo que o gargalo ajustará a velocidade do fluxo dos demais recursos a sua cadência. A 
perda de tempo na restrição será uma perda para o sistema todo, refletindo em uma redução do ganho máximo.

As nove regras do OPT podem auxiliar na sincronização da produção e no resultado global. Para realizar o jogo, uma forma de organizar o sequenciamento e programação é construir uma tabela contendo as operações da máquina restritiva, tempo de setup, tempo de processamento, quantidade à processar e uma coluna contendo o resultado entre a multiplicação da quantidade com o tempo de processamento e a soma do setup. Primeiramente, é necessário balancear a capacidade do posto anterior com base na restrição, sendo a eficiência da restrição que determinará o resultado do posto anterior (Regra 1). É necessário otimizar o sequenciamento e programação para que não ocorra utilização dos recursos não restritivos além do necessário em função da restrição (Regra 2), visando obter máxima eficiência no recurso restritivo, mesmo que isso repercuta em obter menor eficiência em um recurso não restritivo (Regra 3, 4 e 5).

Além disso, os estoques anteriores e posteriores a restrição devem ser otimizados com base na cadência da restrição (Regra 6), e os lotes entre as estações (lote de transferência) podem ser em menor quantidade em detrimento aos lotes processados entre preparações (lote de processamento) (Regra 7), do qual necessitam ser dinâmicos em função das vendas, no caso, inicialmente nas primeiras semanas do jogo, em função das quantidades que garantam caixa para as despesas fixas em cada rodada (Regra 8). O sequenciamento e programação devem ser analisados considerando todas as restrições e/ou informações a serem atendidas, de maneira simultânea (Regra 9). O sequenciamento e programação podem auxiliar a atingir a meta da empresa (RODRIGUES, 1990; BLACKSTONE, 2013).

Por fim, no quarto passo, é organizado o funcionamento entre o recurso restritivo aos demais recursos, concebendo às necessidades e o momento de compras de MP. Este passo é auxiliado pelo TPC. O recurso restritivo dita o ritmo do sistema (tambor), do qual deve ser mantido ininterrupto, para isso, podem serem gerados estoques intermediários que satisfaçam esta necessidade (pulmão), em que são acionadas as necessidades com base no uso pela restrição (corda). No caso do OPT Game, os dados disponibilizados são determinísticos, facilitando o sequenciamento e programação e, ao final do jogo, o objetivo é possuir estoque nulo 
(zero). Em uma empresa, em virtude de variáveis desconhecidas, eventos estocásticos, reforça a necessidade do uso do TPC como meio para auxiliar que o recurso restritivo não pare.

O OPT Game, no final, disponibilizará um relatório financeiro contendo: (i) lado esquerdo, cor "azul": informações do caixa inicial, caixa ao final do jogo, receita com vendas, despesas com MP, despesas operacionais e o fluxo de caixa líquido; (ii) lado esquerdo, cor "magenta": total de produtos vendidos de P, P1 e P2; (iii) lado direito, cor "magenta": resultado da operação das máquinas verde e azul, em percentual acerca do tempo em produção, setup e ocioso. A Figura 2 ilustra estas informações.

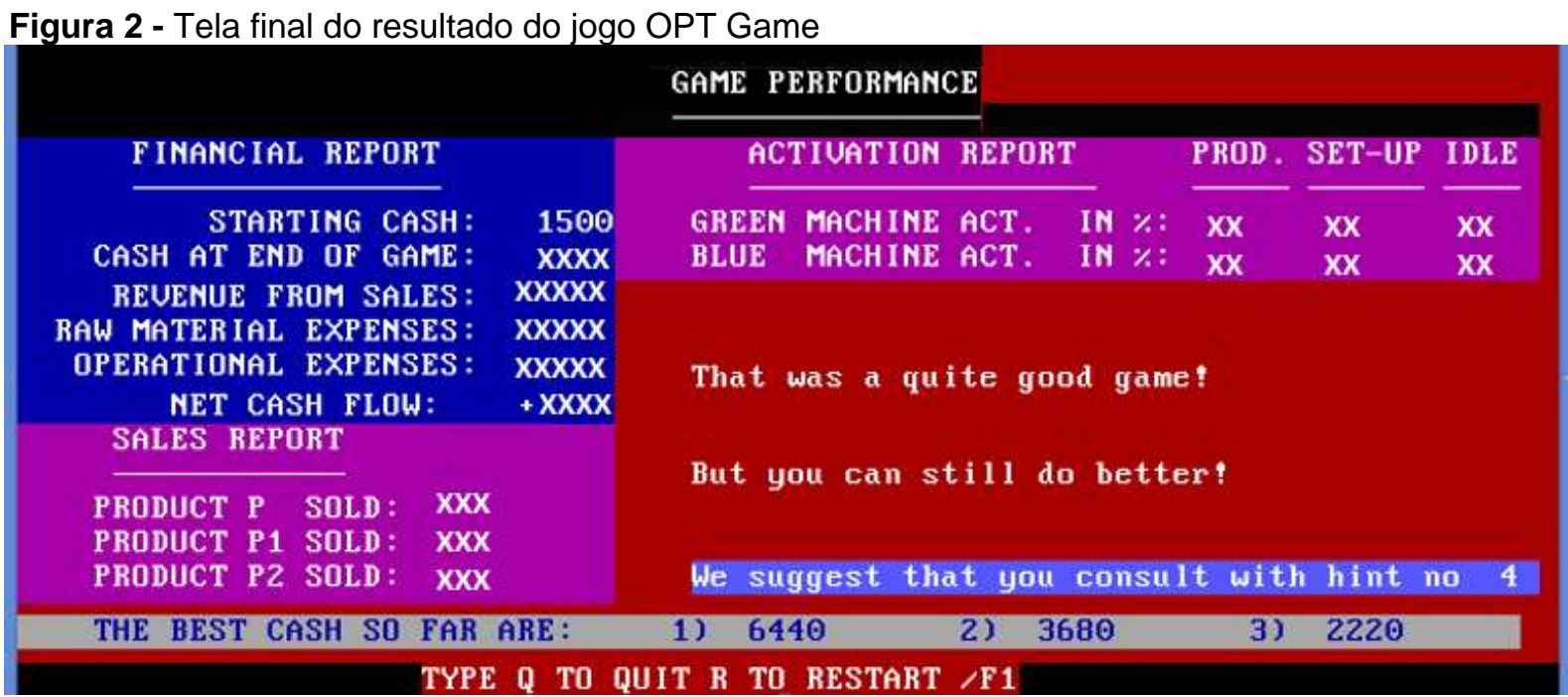

Fonte: adaptado de Muller et al. (1995)

Caso não seja atingido o objetivo ao final das seis semanas, será exibida uma tela com a frase: "você deve ter ido à falência, isso acontece com os melhores de nós". Nesta frase, Goldratt reproduz o que ele imaginava que poderia acontecer com muitas empresas na realidade, quebrariam seu negócio em virtude de não gerir adequadamente suas operações de manufatura. Em se tratando do OPT Game, após a frase citada, Goldratt sugere "entrar em contato com algum consultor", afinal, seus ensinamentos poderiam auxiliar as empresas a obterem melhores resultados e atingir a meta.

\subsection{Estudo Aplicado: resultados e discussões}


O estudo aplicado do OPT Game no formato de aprendizagem ativa buscou desenvolver conhecimentos, entendimento e compreensão de algumas abordagens da TOC. Conforme lógica descrita na seção de metodologia obteve-se os resultados conforme Tabela 3.

Tabela 3 - Resultados de pesquisa

Resultados (\%)
Perguntas

Fonte: Autores (2015)

Os respondentes, em sua maioria, concordam ou concordam plenamente com o uso de jogos no ensino-aprendizagem. Esta reação positiva dos estudantes também foi apresentada quando indagados acerca da motivação em realizar a atividade. Retornos positivos acerca do uso da metodologia ativa são explicitados por alguns autores, citam-se (FERNANDES BRUM; PURCIDONIO; FERRERA, 2017; PONCIANO; GOMES; MORAIS, 2017; ROCHA; LEMOS, 2011; SANTOS; ARNAUD; DUTRA, 2014). A motivação apresentada pelos alunos reforça o objetivo explicitado por Bonwell e Eison (1991) acerca da metodologia ativa em tornar o próprio aluno autônomo. 
Acerca do entendimento e compreensão dos conceitos estudados durante o uso do jogo, percebeu-se que o processo de focalização, o tambor-pulmão-corda e o processo de sincronização foram assimilados pela maior parte dos alunos. Entretanto, referente as regras do OPT e aos indicadores operacionais e globais, ocorreu um menor entendimento. Neste último, percebe-se a necessidade de ajustes, melhorias quanto ao formato do jogo, materiais autoexplicativos ou interferência do mediador, que possibilite ampliar o entendimento dos alunos. Vale destacar que os resultados deste estudo aplicado não contava com auxílio descritivo das etapas, conexões e encadeamento para a execução do jogo. Dispor de mecanismo de auxílio poderia conduzir o aluno à operar o jogo, promover maior entendimento e compreensão.

Este propósito de compreensão vem sendo explorado por alguns autores sob os formatos de dissertações, teses, artigos e livros entre outros meios, citam-se (BOUZADA, 2012; FIGUEIREDO; PEREIRA; VASCONCELLOS, 2013; RIIS, 1995; SCHAFRANSKI, 2002; SCHAFRANSKI; TUBINO, 2013). Estes estudos remetem ao uso da metodologia ativa como forma de elucidar teoria $x$ prática. Segundo as ponderações de Arbex (2005), o uso de jogos possui forte valor pedagógico, proporcionando maior interdisciplinaridade, integração social, competição, cooperação e visão sistêmica.

Por fim, apesar dos relatos positivos das aplicações no ensino em engenharia de produção e isso ter despertado interesse de algumas instituições (SANTOS; ARNAUD; DUTRA, 2014); conforme pesquisa de Pinto et al., (2015), é baixo o número de publicações que contextualizem as aplicações destas metodologias na área.

\section{CONSIDERAÇÕES FINAIS}

O objetivo deste artigo foi apresentar o uso do OPT Game como alternativa para auxiliar no entendimento e compreensão das abordagens da TOC relacionadas ao processo de focalização da TOC, os indicadores globais e indicadores locais, as regras do OPT, sincronização da produção e o tambor-pulmão-corda. Para isso, foi demonstrado por meio de ilustrações e conexões o uso do OPT Game. Por fim, foi apresentado os resultados da aplicação do jogo em uma turma de alunos. 
O OPT Game possibilita que os alunos desenvolvam estratégias em um ambiente fictício que maximize os lucros. Os alunos participam identificando o que restringe o sistema, utilizando algumas abordagens da TOC para identificar o melhor mix de produtos, o quanto produzir e como produzir. Assim como, um ambiente real que permita identificar as relações e conexões nas operações de manufatura que possam influenciar nos resultados.

Em contexto geral, os resultados obtidos com a aplicação do jogo OPT Game inferem positivamente à introdução da metodologia ativa no ensino-aprendizagem na engenharia de produção. Contribui no auxílio a docentes e discentes na contextualização e promoção do entendimento entre teoria e prática.

Por fim, menciona-se a limitação da pesquisa quanto ao tamanho do grupo participante no estudo aplicado. Sugere-se novos estudos que possam complementar esta lacuna. Ademais, o aprimoramento e/ou desenvolvimento de novas simulação acercada da temática, principalmente que possibilitem incluir outras abordagens junto a TOC.

\section{REFERÊNCIAS}

ANTUNES JR, J. Comparação crítica entre os princípios gerais que regem a Teoria das Restrições (TOC) e a teoria que sustenta a construção dos sistemas de produção com estoque zero (SPEZ): no sentido da construção de uma teoria geral das melhorias no processo. In: ENANPAD, 22.,1998. Anais..., 1998.

ANTUNES, José A.V.J. et al. Sistemas de produção: conceitos e práticas para projeto e gestão da produção enxuta. Porto Alegre: Bookman, 2008.

ARBEX, M. A. O valor pedagógico dos jogos de empresa na aprendizagem de gestão de negócios. Revista FAE, v. 8, n. 2, p. 81-89, 2005.

BARBOSA, E.; MOURA, D. Metodologias ativas de aprendizagem na Educação Profissional e Tecnológica. B. Tec. SENAC, v. 39, n.2, p.48-67, 2013.

BLACKSTONE, J. Revisão da literatura sobre tambor-pulmão-corda, gerenciamento de pulmões e distribuição. In: COX III, J.; SCHLEIER, Jr. J. (org.). Handbook da teoria das restrições. Porto Alegre: Bookman, 2013

BONWELL, C.; EISON, J. Active Learning: Creating Excitement in the Classroom, ASHEERIC Higher Education Report No. 1. The George Washington University, School of Education and Human Development. Washington, D.C. 1991.

BOUYER, G.; SZNELWAR, L.; COSTA, M. Aprendizagem estratégica e aquisição conceitual no ensino de gestão de operações. In: ENCONTRO NACIONAL DE ENGENHARIA DE PRODUÇÃO, ABEPRO, 25, 2005. Anais.... Porto Alegre, RS, Brasil., v. 29, 2005. 
BOUZADA, M. A. C. "Jogando" logística no Brasil. Revista de Gestão, v. 19, n. 4, p. 647668, 2012. https://doi.org/10.5700/rege483

BRUNER, Jerome S. The act of discovery. Harvard Educational Review, v.31, p. 21-32, 1961.

CARISSIMI, A. Virtualização: da teoria a soluções. Minicursos do Simpósio Brasileiro de Redes de Computadores - SBRC, 2008. Disponível em:

<http://www.jvasconcellos.com.br/unijorge/wp-content/uploads/2012/01/cap4-v2.pdf>

CARMO, B. B. T. DO; BARROSO, S. H. DE A.; ALBERTIN, M. R. Aprendizagem discente e estratégia docente: metodologias para maximizar o aprendizado no curso de engenharia de produção. Revista Produção Online, v. 10, n. 4, p. 779, 21 nov. 2010.

http://dx.doi.org/10.14488/1676-1901.v10i4.474

COX III, J.; SPENCER Michael. S. Manual da teoria das restrições. Porto Alegre: Bookman, 2008.

FERNANDES BRUM, K.; PURCIDONIO, P. M.; FERREIRA, M. L. Aprendizagem ativa no ensino de engenharia de métodos: uma experiência no CEFET/RJ. Revista Produção Online, v. 17, n. 3, p. 956, 15 set. 2017. http://dx.doi.org/10.14488/1676-1901.v17i3.2739

FIGUEIREDO, Júlio C. B.; PEREIRA, Susana C. F.; VASCONCELLOS, Luis H. R.

Desenvolvimento e Aplicação de um Modelo de Ensino da Teoria das Restrições com Base em uma Abordagem Multiagente. Revista de Administração da Unimep-

Unimep Business Journal, v. 11, n. 1, p. 134-155, 2013.

FIGUEIREDO, J. Modelo Computacional para Simulação de Aplicação da Teoria das Restrições. Revista Alcance, v. 17, n. 2, p. 19-31, 2010.

http://dx.doi.org/10.14210/alcance.v17n2.p019-031

FILHO, Tito Armando Rossi. et al. Aplicação do Processo de Pensamento da Teoria das Restrições para o desenvolvimento sustentável: uma proposição teórica. Revista Espacios. vol. 33, n. 8, 2012.

GOLDRATT, E. A Síndrome do palheiro: garimpando informações num oceano de dados. São Paulo, IMAM, 1991.

GOLDRATT, E. Computerized shop floor scheduling. The International Journal of Production Research, v. 26, n. 3, p. 443-455, 1988.

http://dx.doi.org/10.1080/00207548808947875

GOLDRATT, E. Introdução à TOC: minha visão. In: COX III, JAMES F; SCHLEIER, JR. JOHN G (org.). Handbook da teoria das restrições. Porto Alegre: Bookman, 2013.

GOLDRATT, E.; COX, J. A meta: um processo de melhoria continua. 2. ed. rev. e ampl. São Paulo: Nobel, 2011. 365p.

GUPTA, M.; BOYD, L. Theory of constraints: a theory for operations management. International Journal of Operations \& Production Management, v. 28, n. 10, p. 991-1012, 2008. https://doi.org/10.1108/01443570810903122 
LACERDA, D.; RODRIGUES, L. Analisando algumas ferramentas de Análise de Investimentos à luz da Teoria das Restrições. In: SEGET - SIMPÓSIO DE EXCELÊNCIA EM GESTÃO E TECNOLOGIA, 2007.

MABIN, V.; BALDERSTONE, S. The performance of the theory of constraints methodology: analysis and discussion of successful TOC applications. International Journal of Operations \& Production Management, v. 23, n. 6, p. 568-595, 2003. https://doi.org/10.1108/01443570310476636

MIGUEL, P. A. C. et al. Metodologia de pesquisa em engenharia de produção e gestão de operações. 2. ed. Rio de Janeiro: Elsevier, 2012.

MULLER, H.; BAERTSOEN, G.; VANMAELE, H. Using simulation games within the "CIL_SIM" integrated educational framework for the acquisition of problem solving techniques in industrial management. In: RIIS, J.(org.). Simulation Games and Learning in Production Management. London: Chapman \& Hall, 1995.

PACHECO, D. A., et al. Modelo de gerenciamento da capacidade produtiva: integrando teoria das restrições e o índice de rendimento operacional global (IROG). Revista Produção Online, v. 12, n. 3, p. 806, 13 ago. 2012. http://dx.doi.org/10.14488/1676-1901.v12i3.981

PINTO, C. P. et al. Planejamento, condução e análise do método de avaliação de uma disciplina do curso de engenharia de produção fundamentada na aprendizagem baseada em problemas. Revista Produção Online, v. 15, n. 2, p. 671, 15 jun. 2015.

http://dx.doi.org/10.14488/1676-1901.v15i2.1936

PONCIANO, T. M.; GOMES, F. C. DE V.; MORAIS, I. C. DE. Metodologia ativa na engenharia: verificação da abp em uma disciplina de engenharia de produção e um modelo passo a passo. Revista Principia, n. 34, p. 32-39, 2017.

RIIS, J. O. Simulation Games and learning in production management. London: Schapman \& Hall, 1995.

ROCHA, H. M.; LEMOS, W. D. M. Metodologias Ativas: do que estamos falando? Base conceitual e relato de pesquisa em andamento. IX Simpósio Pedagógico e Pesquisas em Comunicação. Anais...2011.

RODRIGUES, L. H. Apresentação e Análise Crítica da Tecnologia da Produção Otimizada (Optimized Production Technology - OPT) e da Teoria das Restrições (Theory of Constraints - TOC). In: ENCONTRO DA ASSOCIAÇÃO NACIONAL DE PROGRAMAS DE PÓSGRADUAÇÃO EM ADMINISTRAÇÃO, XIV, 1990. Anais.... Florianópolis/SC, 1990.

SANTOS, L. C.; ARNAUD, L. D. M.; DUTRA, J. B. Uma dinâmica para o ensino da matriz produto-processo: roteiro e aplicação. Revista Produção Online, v. 14, n. 3, p. 1129, 18 ago. 2014. http://dx.doi.org/10.14488/1676-1901.v14i3.1700

SCHAFRANSKI, L. E. Jogos de gestão da produção: desenvolvimento e validação. 2002. 195 f.. Tese (Doutorado em Engenharia de Produção) - Universidade Federal de Santa Catarina, Florianópolis, 2002.

SCHAFRANSKI, L. E.; TUBINO, D. F. Simulação Empresarial em Gestão da Produção. São Paulo: Atlas, 2013. 
SOUZA, F. Do OPT à Teoria das Restrições: avanços e mitos. Production Journal, v. 15, n. 2, p. 184-197, 2005. http://dx.doi.org/10.1590/S0103-65132005000200005

SRIKANTH, Mokshagundam. TPC, gerenciamento de pulmões e classificação de fluxo VATI. In: COX III, James F; SCHLEIER, Jr. John G (org.). Handbook da teoria das restrições. Porto Alegre: Bookman, 2013.

VARGAS, E. J., et al. Metodologia tiva de ensino- aprendizagem no ensino de operações de manufatura : O uso do opt game em abordagens da teoria das restrições. In: ENCONTRO NACIONAL DE ENGENHARIA DE PRODUCÃO, 36. 2016. Anais... . João de Pessoa: 2016.

VIGOTSKY, L. Linguagem, desenvolvimento e aprendizagem. 11. ed. São Paulo: Ícne, 2010.

WALS, A. E. J. Sustainability in higher education in the context of the UN DESD: a review of learning and institutionalization processes. Journal of Cleaner Production, v. 62, p. 8-15, jan. 2014. https://doi.org/10.1016/j.jclepro.2013.06.007

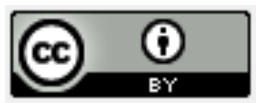

Artigo recebido em 03/03/2017 e aceito para publicação em 19/10/2017

DOI: http://dx.doi.org/10.14488/1676-1901.v18i1.2781 